\title{
A new note on generalized absolute matrix summability
}

\section{HS Özarslan* and T Ari}

\section{"Correspondence:}

seyhan@erciyes.edu.tr

Department of Mathematics, Erciyes

University, Kayseri, 38039, Turkey

\section{Abstract}

This paper gives necessary and sufficient conditions in order that a series $\sum a_{n} \lambda_{n}$ should be summable $|B|_{k}, k \geq 1$, whenever $\sum a_{n}$ is summable $|A|$. Some new results have also been obtained.

MSC: 40D25; 40F05; 40G99

Keywords: summability factors; absolute matrix summability; infinite series

\section{Introduction}

Let $\sum a_{n}$ be a given infinite series with the partial sums $\left(s_{n}\right)$. Let $\left(p_{n}\right)$ be a sequence of positive numbers such that

$$
P_{n}=\sum_{v=0}^{n} p_{v} \rightarrow \infty \quad \text { as }(n \rightarrow \infty), \quad\left(P_{-i}=p_{-i}=0, i \geq 1\right)
$$

The sequence-to-sequence transformation

$$
t_{n}=\frac{1}{P_{n}} \sum_{v=0}^{n} p_{v} s_{v}
$$

defines the sequence $\left(t_{n}\right)$ of the Riesz means of the sequence $\left(s_{n}\right)$ generated by the sequence of coefficients $\left(p_{n}\right)$ (see [3]). The series $\sum a_{n}$ is said to be summable $\left|R, p_{n}\right|_{k}, k \geq 1$, if (see [1])

$$
\sum_{n=1}^{\infty} n^{k-1}\left|t_{n}-t_{n-1}\right|^{k}<\infty
$$

Let $A=\left(a_{n v}\right)$ be a normal matrix, i.e., a lower triangular matrix of nonzero diagonal entries. Then $A$ defines the sequence-to-sequence transformation, mapping the sequence $s=\left(s_{n}\right)$ to $A s=\left(A_{n}(s)\right)$, where

$$
A_{n}(s)=\sum_{v=0}^{n} a_{n v} s_{v}, \quad n=0,1, \ldots
$$


The series $\sum a_{n}$ is said to be summable $|A|_{k}, k \geq 1$, if (see [7])

$$
\sum_{n=1}^{\infty} n^{k-1}\left|\bar{\Delta} A_{n}(s)\right|^{k}<\infty
$$

where

$$
\bar{\Delta} A_{n}(s)=A_{n}(s)-A_{n-1}(s) .
$$

If we take $a_{n v}=\frac{p_{v}}{P_{n}}$, then $|A|_{k}$ summability is the same as $\left|R, p_{n}\right|_{k}$ summability.

Before stating the main theorem we must first introduce some further notations.

Given a normal matrix $A=\left(a_{n v}\right)$, we associate two lover semimatrices $\bar{A}=\left(\bar{a}_{n v}\right)$ and $\hat{A}=\left(\hat{a}_{n v}\right)$ as follows:

$$
\bar{a}_{n v}=\sum_{i=v}^{n} a_{n i}, \quad n, v=0,1, \ldots
$$

and

$$
\hat{a}_{00}=\bar{a}_{00}=a_{00}, \quad \hat{a}_{n v}=\bar{a}_{n v}-\bar{a}_{n-1, v}, \quad n=1,2, \ldots
$$

It may be noted that $\bar{A}$ and $\hat{A}$ are the well-known matrices of series-to-sequence and seriesto-series transformations, respectively. Then, we have

$$
A_{n}(s)=\sum_{\nu=0}^{n} a_{n v} s_{v}=\sum_{\nu=0}^{n} \bar{a}_{n v} a_{v}
$$

and

$$
\bar{\Delta} A_{n}(s)=\sum_{\nu=0}^{n} \hat{a}_{n \nu} a_{\nu} .
$$

If $A$ is a normal matrix, then $A^{\prime}=\left(a_{n v}^{\prime}\right)$ will denote the inverse of $A$. Clearly if $A$ is normal, then $\hat{A}=\left(\hat{a}_{n v}\right)$ is normal and has two-sided inverse $\hat{A}^{\prime}=\left(\hat{a}_{n v}^{\prime}\right)$, which is also normal (see [2]).

Sarıöl [6] has proved the following theorem for $\left|R, p_{n}\right|_{k}$ summability method.

Theorem A Suppose that $\left(p_{n}\right)$ and $\left(q_{n}\right)$ are positive sequences with $P_{n} \rightarrow \infty$ and $Q_{n} \rightarrow \infty$ as $n \rightarrow \infty$. Then $\sum a_{n} \lambda_{n}$ is summable $\left|R, q_{n}\right|_{k}, k \geq 1$ whenever $\sum a_{n}$ is summable $\left|R, p_{n}\right|$, if and only if

$$
\begin{aligned}
& \lambda_{n}=O\left\{n^{\frac{1}{k}-1} \frac{q_{n} P_{n}}{p_{n} Q_{n}}\right\}, \\
& W_{n} \Delta\left(Q_{n-1} \lambda_{n}\right)=O\left(\frac{p_{n}}{P_{n}}\right), \\
& Q_{n} \lambda_{n+1} W_{n}=O(1)
\end{aligned}
$$


provided that

$$
W_{n}=\left\{\sum_{\nu=n+1}^{\infty} v^{k-1}\left(\frac{q_{\nu}}{Q_{\nu} Q_{\nu-1}}\right)^{k}\right\}^{\frac{1}{k}}<\infty .
$$

Theorem B The $\left|R, p_{n}\right|$ summability implies the $\left|R, q_{n}\right|_{k}, k \geq 1$, summability if and only if the following conditions hold:

$$
\begin{aligned}
& \frac{q_{v}}{Q_{v}} \cdot \frac{P_{v}}{p_{v}}=O\left\{v^{\frac{1}{k}-1}\right\}, \\
& \left|\Delta Q_{v-1}\right| \cdot W_{v}=O\left(\frac{p_{v}}{P_{v}}\right), \\
& Q_{v} W_{v}=O(1),
\end{aligned}
$$

where

$$
W_{\nu}=\left\{\sum_{i=v+1}^{\infty} i^{k-1}\left(\frac{q_{i}}{Q_{i} Q_{i-1}}\right)^{k}\right\}^{\frac{1}{k}}<\infty
$$

and we regarded that the above series converges for each $v$ and $\Delta$ is the forward difference operator.

It may be remarked that the above theorem has been proved by Orhan and Sarıgöl [5].

Lemma ([4] $A=\left(a_{n v}\right) \in\left(l_{1}, l_{k}\right)$ if and only if

$$
\sup _{v} \sum_{n=1}^{\infty}\left|a_{n v}\right|^{k}<\infty
$$

for the cases $1 \leq k<\infty$, where $\left(l_{1}, l_{k}\right)$ denotes the set of all matrices $A$ which map $l_{1}$ into $l_{k}=\left\{x=\left(x_{n}\right): \sum\left|x_{n}\right|^{k}<\infty\right\}$.

\section{Main theorem}

The aim of this paper is to generalize Theorem A for the $|A|$ and $|B|_{k}$ summabilities. Therefore we shall prove the following theorem.

Theorem Let $k \geq 1, A=\left(a_{n v}\right)$ and $B=\left(b_{n v}\right)$ be two positive normal matrices such that

$$
\begin{aligned}
& a_{n-1, v} \geq a_{n v}, \quad \text { for } n \geq v+1, \\
& \bar{a}_{n 0}=1, \quad n=0,1,2, \ldots
\end{aligned}
$$

Then, in order that $\sum a_{n} \lambda_{n}$ is summable $|B|_{k}$ whenever $\sum a_{n}$ is summable $|A|$, it is necessary that

$$
\left|\lambda_{n}\right|=O\left(n^{\frac{1}{k}-1} \frac{a_{n n}}{b_{n n}}\right)
$$




$$
\begin{aligned}
& \sum_{n=v+1}^{\infty} n^{k-1}\left|\Delta_{v}\left(\hat{b}_{n v} \lambda_{v}\right)\right|^{k}=O\left(a_{v v}^{k}\right), \\
& \sum_{n=v+1}^{\infty} n^{k-1}\left|\hat{b}_{n, v+1} \lambda_{v+1}\right|^{k}=O(1) .
\end{aligned}
$$

Also (19)-(21) and

$$
\begin{aligned}
& \bar{b}_{n 0}=1, \quad n=0,1,2, \ldots, \\
& a_{n n}-a_{n+1, n}=O\left(a_{n n} a_{n+1, n+1}\right), \\
& \sum_{\nu=r+2}^{\infty}\left|\hat{b}_{n v} \hat{a}_{v r}^{\prime} \lambda_{v}\right|=O\left(\left|\hat{b}_{n, r+1} \lambda_{r+1}\right|\right)
\end{aligned}
$$

are sufficient for the consequent to hold.

It should be noted that if we take $a_{n v}=\frac{p_{v}}{P_{n}}$ and $b_{n v}=\frac{q_{v}}{Q_{n}}$, then we get Theorem A. Also if we take $\lambda_{n}=1$, then we get Theorem $\mathrm{B}$.

\section{Proof of the Theorem}

Necessity. Let $\left(x_{n}\right)$ and $\left(y_{n}\right)$ denote $A$-transform and $B$-transform of the series $\sum a_{n}$ and $\sum a_{n} \lambda_{n}$, respectively. Then, by (8) and (9), we have

$$
\bar{\Delta} x_{n}=\sum_{\nu=0}^{n} \hat{a}_{n \nu} a_{v} \quad \text { and } \quad \bar{\Delta} y_{n}=\sum_{\nu=0}^{n} \hat{b}_{n v} a_{\nu} \lambda_{\nu}
$$

For $k \geq 1$, we define

$$
A=\left\{\left(a_{i}\right): \sum a_{i} \text { is summable }|A|\right\}, \quad B=\left\{\left(a_{i} \lambda_{i}\right): \sum a_{i} \lambda_{i} \text { is summable }|B|_{k}\right\} .
$$

Then it is routine to verify that these are BK-spaces, if normed by

$$
\|X\|=\left\{\sum_{n=0}^{\infty}\left|\bar{\Delta}_{n}\right|\right\}
$$

and

$$
\|Y\|=\left\{\sum_{n=0}^{\infty} n^{k-1}\left|\bar{\Delta} y_{n}\right|^{k}\right\}^{\frac{1}{k}}
$$

respectively. Since $\sum a_{n}$ is summable $|A|$ implies $\sum a_{n} \lambda_{n}$ is summable $|B|_{k}$, by the hypothesis of the theorem,

$$
\|X\|<\infty \quad \Rightarrow \quad\|Y\|<\infty
$$

Now consider the inclusion map $c: A \rightarrow B$ defined by $c(x)=x$. This is continuous, which is immediate as $A$ and $B$ are BK-spaces. Thus there exists a constant $M$ such that

$$
\|Y\| \leq M\|X\|
$$


By applying (25) to $a_{v}=e_{v}-e_{v+1}\left(e_{v}\right.$ is the $v$ th coordinate vector), we have

$$
\bar{\Delta} x_{n}= \begin{cases}0, & \text { if } n<v \\ \hat{a}_{n v}, & \text { if } n=v \\ \Delta_{v} \hat{a}_{n v}, & \text { if } n>v\end{cases}
$$

and

$$
\bar{\Delta} y_{n}= \begin{cases}0, & \text { if } n<v \\ \hat{b}_{n v} \lambda_{v}, & \text { if } n=v \\ \Delta_{v}\left(\hat{b}_{n v} \lambda_{v}\right), & \text { if } n>v\end{cases}
$$

So (26) and (27) give us

$$
\|X\|=\left\{a_{v v}+\sum_{n=v+1}^{\infty}\left|\Delta_{v} \hat{a}_{n v}\right|\right\}
$$

and

$$
\|Y\|=\left\{v^{k-1} b_{v v}\left|\lambda_{v}\right|^{k}+\sum_{n=v+1}^{\infty} n^{k-1}\left|\Delta_{v}\left(\hat{b}_{n v} \lambda_{v}\right)\right|^{k}\right\}^{\frac{1}{k}} .
$$

Hence it follows from (28) that

$$
v^{k-1} b_{\nu v}\left|\lambda_{\nu}\right|^{k}+\sum_{n=v+1}^{\infty} n^{k-1}\left|\Delta_{\nu} \hat{b}_{n v} \lambda_{\nu}\right|^{k} \leq M^{k} a_{\nu v}^{k}+M^{k} \sum_{n=v+1}^{\infty}\left|\Delta_{\nu} \hat{a}_{n v}\right|^{k} .
$$

Using (17), we can find

$$
v^{k-1} b_{v v}\left|\lambda_{v}\right|^{k}+\sum_{n=v+1}^{\infty} n^{k-1}\left|\Delta_{v}\left(\hat{b}_{n v} \lambda_{v}\right)\right|^{k}=O\left(a_{v v}^{k}\right)
$$

The above inequality will be true if and only if each term on the left-hand side is $O\left(a_{v v}^{k}\right)$. Taking the first term,

$$
v^{k-1} b_{v v}\left|\lambda_{v}\right|^{k}=O\left(a_{v v}^{k}\right)
$$

then

$$
\left|\lambda_{v}\right|=O\left(v^{\frac{1}{k}-1} \frac{a_{v v}}{b_{v v}}\right)
$$

which verifies that (19) is necessary. Using the second term, we have

$$
\sum_{n=v+1}^{\infty} n^{k-1}\left|\Delta_{v}\left(\hat{b}_{n v} \lambda_{v}\right)\right|^{k}=O\left(a_{v v}^{k}\right)
$$


which is condition (20). Now, if we apply (22) to $a_{v}=e_{v+1}$, we have

$$
\bar{\Delta} x_{n}= \begin{cases}0, & \text { if } n \leq v \\ \hat{a}_{n, v+1}, & \text { if } n>v\end{cases}
$$

and

$$
\bar{\Delta} y_{n}= \begin{cases}0, & \text { if } n \leq v \\ \hat{b}_{n, v+1} \lambda_{v+1}, & \text { if } n>v\end{cases}
$$

respectively. Hence

$$
\begin{aligned}
& \|X\|=\left\{\sum_{n=v+1}^{\infty}\left|\hat{a}_{n, v+1}\right|\right\}, \\
& \|Y\|=\left\{\sum_{n=v+1}^{\infty} n^{k-1}\left|\hat{b}_{n, v+1} \lambda_{v+1}\right|^{k}\right\}^{\frac{1}{k}} .
\end{aligned}
$$

Hence it follows from (28) that

$$
\sum_{n=v+1}^{\infty} n^{k-1}\left|\hat{b}_{n, v+1} \lambda_{v+1}\right|^{k} \leq M^{k}\left\{\sum_{n=v+1}^{\infty}\left|\hat{a}_{n, v+1}\right|\right\}^{k} .
$$

Using (18) we can find

$$
\sum_{n=v+1}^{\infty} n^{k-1}\left|\hat{b}_{n, v+1} \lambda_{v+1}\right|^{k}=O(1)
$$

which is condition (21).

Sufficiency. We use the notations of necessity. Then

$$
\bar{\Delta} x_{n}=\sum_{v=0}^{n} \hat{a}_{n v} a_{v}
$$

which implies

$$
a_{v}=\sum_{r=0}^{v} \hat{a}_{v r}^{\prime} \bar{\Delta} x_{r}
$$

In this case

$$
\bar{\Delta} y_{n}=\sum_{v=0}^{n} \hat{b}_{n v} a_{v} \lambda_{v}=\sum_{v=0}^{n} \hat{b}_{n v} \lambda_{\nu} \sum_{r=0}^{v} \hat{a}_{v r}^{\prime} \bar{\Delta} x_{r} .
$$

On the other hand, since

$$
\hat{b}_{n 0}=\bar{b}_{n 0}-\bar{b}_{n-1,0}
$$


by (22), we have

$$
\begin{aligned}
\bar{\Delta} y_{n}= & \sum_{v=1}^{n} \hat{b}_{n v} \lambda_{\nu}\left\{\sum_{r=0}^{v} \hat{a}_{v r}^{\prime} \bar{\Delta} x_{r}\right\} \\
= & \sum_{v=1}^{n} \hat{b}_{n v} \lambda_{\nu}\left\{\hat{a}_{v v}^{\prime} \bar{\Delta} x_{v}+\hat{a}_{v, v-1}^{\prime} \bar{\Delta} x_{v-1}+\sum_{r=0}^{v-2} \hat{a}_{v r}^{\prime} \bar{\Delta} x_{r}\right\} \\
= & \sum_{v=1}^{n} \hat{b}_{n v} \lambda_{v} \hat{a}_{v v}^{\prime} \bar{\Delta} x_{v}+\sum_{v=1}^{n} \hat{b}_{n v} \lambda_{v} \hat{a}_{v, v-1}^{\prime} \bar{\Delta} x_{v-1}+\sum_{v=1}^{n} \hat{b}_{n v} \lambda_{v} \sum_{r=0}^{v-2} \hat{a}_{v r}^{\prime} \bar{\Delta} x_{r} \\
= & \hat{b}_{n n} \lambda_{n} \hat{a}_{n n}^{\prime} \bar{\Delta} x_{n}+\sum_{v=1}^{n-1}\left(\hat{b}_{n v} \lambda_{\nu} \hat{a}_{v v}^{\prime}+\hat{b}_{n, v+1} \lambda_{v+1} \hat{a}_{v+1, v}^{\prime}\right) \bar{\Delta} x_{v} \\
& +\sum_{r=0}^{n-2} \bar{\Delta} x_{r} \sum_{v=r+2}^{n} \hat{b}_{n v} \lambda_{v} \hat{a}_{v r}^{\prime} .
\end{aligned}
$$

By considering the equality

$$
\sum_{k=v}^{n} \hat{a}_{n k}^{\prime} \hat{a}_{k v}=\delta_{n v}
$$

where $\delta_{n v}$ is the Kronecker delta, we have that

$$
\begin{aligned}
\hat{b}_{n v} \lambda_{v} \hat{a}_{v v}^{\prime}+\hat{b}_{n, v+1} \lambda_{v+1} \hat{a}_{v+1, v}^{\prime} & =\frac{\hat{b}_{n v} \lambda_{v}}{\hat{a}_{v v}}+\hat{b}_{n, v+1} \lambda_{v+1}\left(-\frac{\hat{a}_{v+1, v}}{\hat{a}_{v v} \hat{a}_{v+1, v+1}}\right) \\
& =\frac{\hat{b}_{n v} \lambda_{v}}{a_{v v}}-\frac{\hat{b}_{n, v+1} \lambda_{v+1}\left(\bar{a}_{v+1, v}-\bar{a}_{v, v}\right)}{a_{v v} a_{v+1, v+1}} \\
& =\frac{\hat{b}_{n v} \lambda_{v}}{a_{v v}}-\frac{\hat{b}_{n, v+1} \lambda_{v+1}\left(a_{v+1, v+1}+a_{v+1, v}-a_{v v}\right)}{a_{v v} a_{v+1, v+1}} \\
& =\frac{\Delta_{v}\left(\hat{b}_{n v} \lambda_{v}\right)}{a_{v v}}+\hat{b}_{n, v+1} \lambda_{v+1} \frac{a_{v v}-a_{v+1, v}}{a_{v v} a_{v+1, v+1}}
\end{aligned}
$$

and so

$$
\begin{aligned}
\bar{\Delta} y_{n}= & \frac{b_{n n} \lambda_{n}}{a_{n n}} \bar{\Delta} x_{n}+\sum_{v=1}^{n-1} \frac{\Delta_{v}\left(\hat{b}_{n v} \lambda_{v}\right)}{a_{v v}} \bar{\Delta} x_{v}+\sum_{v=1}^{n-1} \hat{b}_{n, v+1} \lambda_{v+1} \frac{a_{v v}-a_{v+1, v}}{a_{v v} a_{v+1, v+1}} \bar{\Delta} x_{v} \\
& +\sum_{r=0}^{n-2} \bar{\Delta} x_{r} \sum_{v=r+2}^{n} \hat{b}_{n v} \lambda_{v} \hat{a}_{v r}^{\prime} .
\end{aligned}
$$

Let

$$
\begin{aligned}
& T_{n}(1)=\frac{b_{n n} \lambda_{n}}{a_{n n}} \bar{\Delta} x_{n}+\sum_{\nu=1}^{n-1} \frac{\Delta_{v}\left(\hat{b}_{n v} \lambda_{v}\right)}{a_{v v}} \bar{\Delta} x_{v}+\sum_{v=1}^{n-1} \hat{b}_{n, v+1} \lambda_{v+1} \frac{a_{v v}-a_{v+1, v}}{a_{v v} a_{v+1, v+1}} \bar{\Delta} x_{v}, \\
& T_{n}(2)=\sum_{r=0}^{n-2} \bar{\Delta} x_{r} \sum_{\nu=r+2}^{n} \hat{b}_{n v} \lambda_{\nu} \hat{a}_{v r}^{\prime} .
\end{aligned}
$$


Since

$$
\left|T_{n}(1)+T_{n}(2)\right|^{k} \leq 2^{k}\left(\left|T_{n}(1)\right|^{k}+\left|T_{n}(2)\right|^{k}\right)
$$

to complete the proof of Theorem, it is sufficient to show that

$$
\sum_{n=1}^{\infty} n^{k-1}\left|T_{n}(i)\right|^{k}<\infty \quad \text { for } i=1,2
$$

Then

$$
\begin{aligned}
\overline{T_{n}(1)} & =n^{1-\frac{1}{k}} T_{n}(1) \\
& =n^{1-\frac{1}{k}} \frac{b_{n n} \lambda_{n}}{a_{n n}} \bar{\Delta} x_{n}+n^{1-\frac{1}{k}} \sum_{v=1}^{n-1} \frac{\Delta_{v}\left(\hat{b}_{n v} \lambda_{v}\right)}{a_{v v}} \bar{\Delta} x_{v}+n^{1-\frac{1}{k}} \sum_{v=1}^{n-1} \hat{b}_{n, v+1} \lambda_{v+1} \frac{a_{v v}-a_{v+1, v}}{a_{v v} a_{v+1, v+1}} \bar{\Delta} x_{v} \\
& =\sum_{v=1}^{\infty} c_{n v} \bar{\Delta} x_{v},
\end{aligned}
$$

where

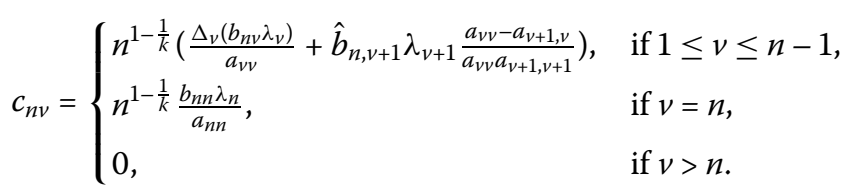

Now

$$
\sum\left|\overline{T_{n}(1)}\right|^{k}<\infty \quad \text { whenever } \sum\left|\bar{\Delta} x_{n}\right|<\infty
$$

is equivalently

$$
\sup _{v} \sum_{n=1}^{\infty}\left|c_{n v}\right|^{k}<\infty
$$

by Lemma. But it follows from conditions (20), (21) and (23) that

$$
\begin{aligned}
\sum_{n=v}^{\infty}\left|c_{n v}\right|^{k} & =O(1)\left\{n^{k-1}\left|\frac{b_{n n} \lambda_{n}}{a_{n n}}\right|^{k}+\sum_{n=v+1}^{\infty} n^{k-1}\left|\frac{\Delta_{\nu}\left(\hat{b}_{n \nu} \lambda_{\nu}\right)}{a_{\nu v}}+\hat{b}_{n, v+1} \lambda_{\nu+1} \frac{a_{\nu v}-a_{\nu+1, v}}{a_{\nu v} a_{\nu+1, v+1}}\right|^{k}\right\} \\
& =O(1) \quad \text { as } v \rightarrow \infty .
\end{aligned}
$$

Finally,

$$
\overline{T_{n}(2)}=n^{1-\frac{1}{k}} T_{n}(2)=n^{1-\frac{1}{k}} \sum_{r=0}^{n-2} \bar{\Delta} x_{r} \sum_{v=r+2}^{n} \hat{b}_{n v} \hat{a}_{v r}^{\prime} \lambda_{v}=\sum_{r=0}^{\infty} d_{n r} \bar{\Delta} x_{r},
$$

where

$$
d_{n r}= \begin{cases}n^{1-\frac{1}{k}} \sum_{v=r+2}^{n} \hat{b}_{n v} \hat{a}_{v r}^{\prime} \lambda_{v}, & \text { if } 0 \leq r \leq n-2, \\ 0, & \text { if } r>n-2 .\end{cases}
$$


Now

$$
\sum\left|\overline{T_{n}(2)}\right|^{k}<\infty \quad \text { whenever } \sum\left|\bar{\Delta} x_{n}\right|<\infty
$$

is equivalently

$$
\sup _{r} \sum_{n=1}^{\infty}\left|d_{n r}\right|^{k}<\infty
$$

by Lemma. But it follows from conditions (21) and (24) that

$$
\begin{aligned}
\sum_{n=r+2}^{\infty}\left|d_{n r}\right|^{k} & =O(1) \sum_{n=r+2}^{\infty} n^{k-1}\left\{\sum_{v=r+2}^{\infty}\left|\hat{b}_{n v} \hat{a}_{v r}^{\prime} \lambda_{v}\right|\right\}^{k} \\
& =O(1) \sum_{n=r+2}^{\infty} n^{k-1}\left|\hat{b}_{n, r+1} \lambda_{r+1}\right|^{k} \\
& =O(1) \text { as } r \rightarrow \infty .
\end{aligned}
$$

Therefore, we have

$$
\sum_{n=1}^{\infty} n^{k-1}\left|T_{n}(i)\right|^{k}<\infty \quad \text { for } i=1,2
$$

This completes the proof of the Theorem.

Received: 3 June 2012 Accepted: 13 July 2012 Published: 27 July 2012

\section{References}

1. Bor, H: On the relative strength of two absolute summability methods. Proc. Am. Math. Soc. 113, 1009-1012 (1991)

2. Cooke, RG: Infinite Matrices and Sequence Spaces. Macmillan \& Co., London (1950)

3. Hardy, GH: Divergent Series. Oxford University Press, Oxford (1949)

4. Maddox, IJ: Elements of Functional Analysis. Cambridge University Press, Cambridge (1970)

5. Orhan, C, Sarıgöl, MA: On absolute weighted mean summability. Rocky Mt. J. Math. 23(3), 1091-1098 (1993)

6. Sarıgöl, MA: On the absolute Riesz summability factors of infinite series. Indian J. Pure Appl. Math. 23(12), 881-886 (1992)

7. Tanovič-Miller, N: On strong summability. Glas. Mat. 34, 87-97 (1979)

doi:10.1186/1029-242X-2012-166

Cite this article as: Özarslan and Ari: A new note on generalized absolute matrix summability. Journal of Inequalities and Applications 2012 2012:166. 